\title{
Recherches qualitatives
}

\section{De la (re)découverte des affects à l'affectivité limitée : une ethnographie enrichie par la combinaison méthodologique}

Thomas Bonnet et Amandine Rochedy

Volume 39, numéro 2, automne 2020

Enquêter sur les affects : quels enjeux, quelles méthodes ?

URI : https://id.erudit.org/iderudit/1073506ar

DOI : https://doi.org/10.7202/1073506ar

Aller au sommaire du numéro

Éditeur(s)

Association pour la recherche qualitative (ARQ)

ISSN

1715-8702 (numérique)

Découvrir la revue

Citer ce document

Bonnet, T. \& Rochedy, A. (2020). De la (re)découverte des affects à l'affectivité

limitée : une ethnographie enrichie par la combinaison méthodologique.

Recherches qualitatives, 39(2), 1-14. https://doi.org/10.7202/1073506ar d'utilisation que vous pouvez consulter en ligne. 


\section{Introduction}

\section{De la (re)découverte des affects à l'affectivité limitée : une ethnographie enrichie par la combinaison méthodologique}

Thomas Bonnet, Ph. D.

Université Lyon 2, France

Amandine Rochedy, Ph. D.

Université Toulouse Jean Jaurès, France

Ce numéro de Recherches qualitatives contribue à saisir les enjeux et à identifier les méthodes pour enquêter sur les affects ${ }^{1}$. Il aborde la dimension affective de l'enquête selon une triple perspective : en tant qu'objet d'étude avec les enjeux qui y sont liés, comme une ressource dans l'analyse des données, mais également dans ses aspects méthodologiques. Cette introduction vise certes à présenter les articles composant ce numéro, mais également à poser quelques éléments nécessaires à la compréhension de la problématique de l'ethnographie des affects.

\section{La (re)découverte des affects dans les sciences humaines et sociales}

Les recherches en sciences humaines et sociales s'intéressant à la question affective ont connu depuis la fin $\mathrm{du} \mathrm{XX}^{\mathrm{e}}$ siècle un intérêt considérable au point que d'aucuns évoquent un «tournant affectif» (Clough \& Halley, 2007). Le propos mérite d'être cependant nuancé, car ce nouveau parti pris d'utiliser les affects comme objet de recherche ou catégorie d'analyse diverge d'une discipline à l'autre. Si la psychologie ou la philosophie ont instauré ce mouvement très tôt, d'autres sciences l'ont suivi plus récemment, mais de manière appuyée comme l'histoire par exemple. Des historiens mobilisent aujourd'hui les affects en tant que «mode historiographique » (Deluermoz et al., 2013) répondant ainsi favorablement au lointain appel de Febvre (1941) qui plaidait jadis pour une histoire des sensibilités. En témoigne par exemple

RECHERCHES QUALITATIVES - Vol. 39(2), pp. 1-14.

ENQUÊTER SUR LES AFFECTS : QUELS ENJEUX, QUELLES MÉTHODES?

ISSN 1715-8702 - http://www.recherche-qualitative.qc.ca/revue/

(C) 2020 Association pour la recherche qualitative 
l'entreprise dirigée par certains d'entre eux de retracer en trois volumes l'Histoire des émotions (Corbin et al., 2016a, 2016b, 2017). D'autres disciplines, comme la sociologie ou la science politique, procèdent plutôt à une réhabilitation scientifique de l'objet affectif. Il convient de voir en effet que les affects n'étaient pas absents des recherches réalisées par les «fondateurs » de la sociologie à la fin du XIX ${ }^{\mathrm{e}}$ siècle, tels Durkheim ou Weber (Bernard, 2014; Cuin, 2001). En revanche, les affects étaient d'emblée estimés comme s'opposant à la rationalité. Dans la mesure où l'étude de la rationalité et des sociétés modernes constituaient l'objet de la sociologie d'alors (Mendras \& Étienne, 1996), les affects renvoyaient, eux, au statut de la primitivité. Ils étaient en quelque sorte le contre-exemple de l'objet sociologique. La science politique, à l'instar de la sociologie, a pendant longtemps fait preuve de méfiance à l'égard des affects, notamment dans l'explication des mobilisations collectives. Cette prise de distance tenait pour beaucoup à la crainte de voir le chercheur étudiant les affects comme dimension dans l'engagement politique être rattaché au courant de la psychologie des foules (Traïni \& Siméant, 2009). Cette approche, considérant l'affect comme relevant du pathos et totalement subjectif, s'opposait alors aux canons du positivisme.

Le tournant affectif s'explique vraisemblablement par au moins deux phénomènes. D'une part, un socle de travaux précurseurs qui avaient opéré le choix de se pencher sur la question affective sans utiliser les affects comme antithèse à la rationalité. On peut penser, entre autres, à des recherches sur l'expression obligatoire des sentiments (Mauss, 1921), la socialisation des émotions en science de l'éducation (Montandon, 1992), leurs usages en politique (Braud, 1996) ou encore leur marchandisation dans les mondes du travail (Hochschild, 1983). D'autre part, les travaux de Damasio (1995) et de Ledoux (2005) dans le domaine des neurosciences, au milieu des années 1990, ont permis de mieux comprendre comment les affects jouaient un rôle fondamental dans la prise de décision raisonnée. Ce double mouvement a participé à la légitimation de l'étude des affects dans les sciences humaines et sociales en ouvrant la voie à un engouement et à une redécouverte de cet objet. Cependant, cet apport entre le «neural» et le «social» ne s'est pas fait sans controverses scientifiques, démontrant ainsi les tensions politiques et sociales sous-jacentes à cette question (Deluermoz et al., 2018). En outre, les apports mutuels ne sont pas non plus équivalents, les sciences humaines et sociales s'appuyant nettement plus sur l'apport des neurosciences que l'inverse (Ehrenberg, 2018). 


\section{Les affects : objet de recherche et catégorie d'analyse légitime?}

La légitimité de mobiliser les affects comme un objet de recherche ou une catégorie d'analyse mérite d'être posée. Sans pouvoir instruire cette problématique épistémologique sur le terrain de l'ensemble des disciplines qui composent les sciences humaines et sociales, nous pouvons néanmoins souligner que les diverses contributions pluridisciplinaires regroupées dans ce numéro la posent en filigrane ou de manière explicite. La question de la légitimité est souvent appréhendée comme démarche méthodologique pertinente pour ethnographier les affects : c'est l'objet même du numéro. Ainsi, le lecteur pourra découvrir des approches variées relevant de traditions disciplinaires diverses, alors même que toutes s'inscrivent dans l'approche ethnographique. L'entretien ou le récit de vie, techniques de verbalisation des affects éprouvés, apparaissent dans la très grande majorité des contributions. Mais aucune d'entre elles ne se limite pour autant à cela. En complément à la technique de l'entretien, plusieurs auteurs - Gobatto, Loriol, Tcholakova, Vasquez et Yadan - utilisent l'observation consignée sur carnets alors que d'autres - Ocquidant - filment leurs observations. Ces dernières peuvent ainsi être à l'origine d'entretiens sur l'activité des enquêtés à travers l'autoconfrontation simple ou croisée, respectivement employée par Pham Quang et Poussin. Ce sont dans tous les cas systématiquement des méthodes combinées ou complexes qui sont utilisées. Le travail sur archives audiovisuelles couplé à l'usage d'un logiciel d'analyse textuelle, développé par Catlla, ou la combinaison de récits de vie et de calendriers de vie par Sommier souligne la nécessité d'armer une méthodologie propre à l'ethnographie des affects. Ce besoin s'exprime nettement durant les moments de réflexivité des contributeurs ou lors de la formation d'apprentis ethnographes comme le développent Perriard et ses collègues.

Une méthodologie simple semble insuffisante pour ethnographier les affects et demande souvent de faire converger différentes méthodes. S'il faut déceler l'affect dans les mots de l'autre, en l'interrogeant et en les consignant à l'écrit ou numériquement, il semble nécessaire d'aller plus loin : prendre en compte la tonalité de la voix, les gestes et les postures du corps, les expressions faciales ou le regard des enquêtés. Cette attention permet de saisir l'expression émotionnelle du social. Mais, elle oblige le chercheur à opérer un travail réflexif. Certes, comme nous venons de le souligner, cette réflexivité peut porter sur les choix opérés dans la méthode pour ethnographier au mieux les affects. Cependant, les questionnements portent aussi sur la façon d'étudier et d'écrire sur un objet à la légitimité quelquefois encore "douteuse ». Enfin, c'est éminemment les effets des affects qui sont sujets à la démarche réflexive. 
Si le chercheur peut s'interroger sur ce que le terrain lui fait, il gagne également à penser à ce qu'il fait au terrain : qu'a pu déclencher le chercheur chez les enquêtés par son travail? Ethnographier les affects, c'est prendre potentiellement le risque d'affecter les sujets. Dès lors, jusqu'où aller? La réponse n'est pas simple; elle engage l'éthique de l'ethnographe et plusieurs voies se dessinent entre deux pôles ${ }^{2}$.

D'un côté, celui où le chercheur se méfierait viscéralement de la dimension affective du social. Souscrivant à l'indépassable rupture épistémologique (Durkheim, 1894) ou poursuivant l'idéal de l'absolue neutralité axiologique (Weber, 1917/1965), ce chercheur adopte une posture surplombante à l'égard des affects. Nous pensons là qu'il s'agit d'une position intenable parce que chimérique et qu'elle soumet son adepte à des aveuglements importants. D'un autre côté, l'enquêteur peut tant reconnaître les affects des enquêtés qu'il s'estime être redevable d'une dette morale à leur égard (Villani et al., 2014). Cette posture pointe un écueil majeur dans le risque que le chercheur (auto)investit d'une charge affective, ne fasse que rapporter les sentiments de la population enquêtée, sans pour autant les passer au crible de l'analyse.

Comme le souligne Bernard (2017), reprenant les réflexions d'Elias (1983/1993), il s'agit d'opérer à partir d'un continuum entre engagement et distanciation. Il ne s'agit pas tant de tracer une troisième voie que de souligner l'amplitude des possibles. En premier lieu, considérer une distance juste parce qu'elle se situe au milieu n'a aucun sens, puisque le «juste» milieu diffère entre chaque chercheur ethnographiant les affects. Des différences interindividuelles existent en lien avec l'histoire personnelle du chercheur, dans son rapport à l'objet, dans son expérience de terrain et des traditions méthodologiques et épistémologiques qu'il mobilise. Chacun de ces paramètres peut permettre à l'enquêteur d'étalonner ce qui semble être la «bonne» ou la « juste » distance. Cela peut être, finalement, un accord trouvé entre son terrain et sa discipline et qui bien sûr le satisfasse, qui légitime sa façon d'ethnographier les affects. Dès lors qu'une place suffisamment importante est accordée dans la recherche pour expliciter sa posture, la prise de distance ou l'engagement envers l'objet de recherche peut se comprendre. Dans tous les cas, en se donnant à voir, cette préoccupation permet au lecteur de saisir la place du chercheur et le rôle qu'il a adopté. Ce faisant, le lecteur peut se positionner sur un des aspects importants de la mécanique interne de production de la recherche : pourquoi le chercheur s'estime-t-il engagé dans un contre-don émotionnel à l'égard des enquêtés? Comment peut-il, à l'inverse, faire montre d'un tel détachement envers son terrain? On retrouve alors là de vieux préceptes sur la nécessité d'une prise de distance avec son objet, tout en 
permettant de faire apparaître l'engagement du chercheur envers le matériau sensible qu'il a pu recueillir et qu'il a pu aussi produire. Mais ce travail n'est pas facile à mener et nombre de ceux qui ont contribué à ce numéro évoquent ces difficultés avec mérite.

\section{De la difficulté à appréhender les affects dans la recherche qualitative à l'« affectivité limitée »}

Le terme mérite n'est pas excessif. Il tient au fait, selon nous, qu'il est, comme le souligne Bizeul (2007), extrêmement tentant de passer sous silence, lors de la restitution de la recherche, les épisodes «peu flatteurs » de celle-ci. Or les difficultés rapportées par les contributions sur la place des émotions dans la relation d'enquête permettent de saisir un enjeu important dans cet exercice. Nous savons à quel point la position de l'observateur doit être interrogée pour comprendre la situation observée (Devereux, 1967/1980). Nous savons aussi combien les phases de réflexivité apportent à l'objet de recherche en question, mais, plus généralement, à la pratique d'une discipline, notamment quand elles sont fondées sur ce qui n'a pas marché (Kuhn, 2018). Mais si l'on tient compte de la perspective d'être accusé d'égocentrisme en usant du «"je" méthodologique » (Olivier de Sardan, 2000), nous mesurons le mérite qu'il faut pour évoquer ces limites de la recherche. Ethnographier les affects et communiquer à ce sujet dans des réseaux académiques, c'est encore aujourd'hui prendre le risque de se retrouver taxé d'un subjectivisme rampant. Cette mise à l'index de ce que fait émotionnellement la recherche au chercheur et de ce que ce dernier fait émotionnellement à la recherche est dommageable pour nos disciplines.

Plutôt que de s'obstiner à croire que parler d'affect dans la recherche consiste à autocentrer le regard du chercheur sur sa sentimentalité, il s'agit d'effectuer la démarche opposée : s'interroger sur les affects dans la recherche, c'est en quelque sorte procéder d'une manière parallèle à la réflexion sur les limites de la rationalité du chercheur. À l'instar de la «rationalité limitée » théorisée en 1958 par les économistes March et Simon (1958/1999) et qui montre les limites des dimensions cognitives du comportement de l'acteur, le chercheur, comme tout être humain, est sujet à une affectivité limitée. Celle-ci est bornée pour l'essentiel par des cadres sociaux qui la normalisent et par la nécessité d'agir dans la situation qui se présente à lui avec les éléments, entre autres affectifs, qu'il a à sa disposition. Dès lors, opérer un travail de réflexivité affective permet d'adjoindre au protocole de recherche une compréhension des limites de l'affectivité du chercheur et de la dimension affective de la recherche. Cette démarche non seulement favorise la transparence de l'ethnographie déployée, renforce sa légitimité et procède d'une tentative 
d'objectivation de la posture d'enquête (Ghasarian, 1997). Qui plus est, en engageant le chercheur à un rigoureux examen des possibilités affectives, cette démarche oblige à une humilité qui signe plus une ouverture à la critique qu'un repli sur soi.

Par ailleurs, le mérite qui émane des contributions présentes dans ce numéro est très fortement lié à l'absence, due à un oubli ou à un silence, dont font souvent preuve les formations initiales des ethnographes sur la question du comment composer sur le terrain avec nos affects et ceux des personnes enquêtées (Peneff, 2009). Les ethnographes sont en effet bien souvent livrés à eux-mêmes et aux conseils trouvés çà et là pour éviter les multiples écueils de l'ethnographie des affects. Gageons que les contributions présentes dans ce numéro permettront un apport en la matière et une aide dans la réalisation d'enquête.

\section{Présentation des contributions}

Le numéro regroupe les résultats de recherches empiriques menées par des chercheurs de disciplines différentes. En plus de favoriser les travaux pluridisciplinaires, le dossier a valorisé des articles issus de la recherche francophone dont les auteurs jouissent de statuts institutionnels différents. Les onze contributions présentées traitent de terrains hétérogènes (milieu scolaire, sphères politiques, situations de soin, espaces urbains, mondes du travail, etc.), auprès de populations plurielles (professionnels, militants, migrants, parents, etc.).

Isabelle Gobatto se demande «sous quelles modalités méthodologiques et avec quels effets peut-on utiliser ses affects en tant qu'anthropologue pour conduire une recherche et, en particulier, pour rendre compte des émotions des personnes avec lesquelles on interagit? ». Ce questionnement méthodologique s'inscrit dans un contexte de pauvreté à travers plusieurs recherches ethnographiques lors de situations de soins (prévention du VIH de la mère à l'enfant, maladie chronique, etc.) en Afrique de l'Ouest où une multitude d'émotions sont présentes lors de l'enquête ethnographique. Son analyse rétrospective et réflexive des dimensions émotionnelles des enquêtés et de l'enquêteur dans le processus d'enquête participe à l'intelligence des situations. Le déplacement de la question affective depuis ses dimensions méthodologiques vers l'éthique sert pour décrire et comprendre le contexte social dans lequel sont délivrés les soins et pour favoriser l'échange dans l'enquête. Mais, à l'inverse, Isabelle Gobatto souligne également comment l'appréhension de la dimension des affects perturbe le travail ethnographique.

Marc Loriol s'intéresse à la question de la coloration affective dans deux milieux professionnels spécifiques : celui des diplomates et des policiers 
de voie publique. L'auteur défend l'idée qu'à l'inverse des expériences en laboratoire sur les émotions, il est indispensable de prendre en compte la complexité du contexte social et des interactions qui s'y déroulent. Cela confère alors à la méthode ethnographique toute son importance pour enquêter sur les affects. Cette ethnographie permet au chercheur d'accéder à la façon dont les professionnels, en collectif ou individuellement, colorent les épisodes émotionnels vécus. Pour saisir comment le travail est affectivement teinté, l'immersion apparait pour Marc Loriol comme le moyen de voir comment le collectif puise dans la grammaire affective et culturelle du milieu enquêté pour nuancer les épisodes vécus. Cependant, Marc Loriol s'interroge sur les effets potentiels de cette immersion: «Est-ce que je ne projette pas indûment les émotions que je ressens sur les personnes observées? Est-ce que mes propres émotions ne risquent pas de déformer mon jugement sur ce que j'observe? » Son ressenti questionné à l'aune de celui des enquêtés et des codes socioémotionnels qu'il décrit se constitue comme un bon indicateur pour poursuivre ou réajuster l'enquête. Plus qu'un moyen pour ethnographier les affects, la coloration affective est un outil permettant d'associer diverses approches disciplinaires.

En se penchant sur les expressions émotionnelles des candidats malheureux le soir du second tour des élections présidentielles de la cinquième République française (1965-2017), Michel Catlla propose de voir comment celles-ci renseignent sur les normes émotionnelles de cet exercice et sur les codes émotionnels en vigueur à ces différentes époques. En s'appuyant sur l'étude d'archives et en utilisant l'analyse textuelle à l'aide du logiciel Iramuteq, l'auteur dégage trois postures émotionnelles adoptées par les vaincus se prêtant à l'exercice d'un discours public. Il montre les différences de styles émotionnels, témoignant finalement de l'évolution des normes émotionnelles. Ainsi, les défaites aux débuts de la cinquième République se caractérisent par une sobriété, un « ascétisme émotionnel ». Les discours des années 1980, quant à eux, s'inscrivent dans «l'exemplarité émotionnelle». Enfin, la défaite électorale des trois dernières décennies s'exprime comme un «événement émotionnel », une sorte de communion auprès des fidèles. In fine, de l'analyse textuelle et de l'étude du langage non verbal de cet ensemble de discours de l'insuccès présidentiel, Michel Catlla dégage deux types de familles d'émotions récurrentes : d'un côté des émotions attendues s'inscrivant dans le rituel du jeu politique, de l'autre des émotions visant à enrôler l'auditoire et à préparer les prochaines échéances politiques.

L'article d'Isabelle Sommier part d'un constat: il est extrêmement difficile aujourd'hui de saisir les carrières affectives des militants faute d'une méthodologie adéquate. Proposant un regard réflexif sur une recherche 
collective portant sur la carrière de militants des années 1968 (l'enquête Sombrero [Sociologie du militantisme, biographies, réseaux, organisations]), à laquelle a participé l'auteure, cette dernière nous livre ses réflexions sur une façon possible de résoudre ce manque. Les quelque 400 récits de vie associés aux 285 calendriers de vie permettent certes de saisir l'articulation du temps biographique et des temps sociaux dans l'engagement militant et, ce faisant, enrichissent la compréhension des identités du militantisme, mais les ressorts affectifs de l'engagement ou du désengagement semblent des points aveugles. Après avoir présenté les apports et les limites de l'approche biographique mobilisée pour l'enquête, Isabelle Sommier souligne combien la prise en compte de certaines pistes pourrait être fructueuses, comme le travail à partir d'archives ou l'usage de la vidéo par exemple.

Long Pham Quang aborde justement dans sa contribution l'intérêt de la vidéo pour ethnographier les « émotions occurrentes ». L'auteur s'intéresse à la place que jouent les émotions dans la relation entre un stagiaire et son tuteur durant l'apprentissage de soins mortuaires. Plus que saisir le climat émotionnel de ces situations de travail, l'article montre comment les émotions vécues affectent la formation des agents. Le dispositif déployé et analysé dans cet article est un triptyque méthodologique. D'abord, le chercheur observe et identifie les situations émotionnellement pertinentes pour sa recherche. Par la suite, il tente de saisir les affects de la situation d'apprentissage par la vidéo. Enfin, il procède à des autoconfrontations pour expliciter les images enregistrées. Long Pham Quang mobilise deux exemples pour illustrer les apports d'un tel dispositif. Au-delà de cette contribution à l'apport méthodologique pour ethnographier les affects, l'auteur pose également un regard réflexif sur la façon dont ses propres affects ont influencé la méthodologie déployée.

Olivier Ocquidant propose aussi d'enquêter les affects à travers l'image. Comment saisir par une approche ethnographique sensible les espaces urbains? Le postulat du chercheur est que pour comprendre la ville, il faut y déambuler. En prenant l'exemple d'une marche dans la ville de Saint-Étienne, l'auteur développe son propos à partir d'une double approche. La première relève d'une observation dynamique (même si l'observation statique n'est pas exclue) à laquelle se réfère Olivier Ocquidant pour comprendre les espaces sensibles. Ce dispositif de marche à la première personne, qui peut aussi se décliner au pluriel, consiste à accompagner les enquêtés durant leur marche. Le recueil de la parole devient alors important et plutôt qu'une observation participante, c'est en quelque sorte ce que nous pourrions appeler une observation dialoguante à laquelle procède le sociologue. La méthodologie présentée dans cette contribution s'enrichit au fil de la lecture d'une réflexion 
sur l'usage de la photographie et de la vidéo pour comprendre les types d'engagements qui se déroulent dans l'espace public. Olivier Ocquidant présente alors les avantages et les inconvénients tant de la marche filmée que des plans vidéos fixes.

La contribution de Nadine Poussin propose de s'intéresser à l'ethnographie des affects à partir d'une intervention en psychologie du travail auprès de médecins du travail. Mobilisant le cadre théorique de la clinique de l'activité, l'auteure interroge l'apport méthodologique de l'autoconfrontation croisée filmée. Pour ce faire, elle souligne l'intérêt d'exploiter à partir du matériau vidéo recueilli des modalités particulières, à savoir le regard, la voix et les mots des enquêtés filmés. Que se passe-t-il quand à certains moments d'échanges la situation paraît perturbée? Comment cette analyse multimodale permet-elle de pousser la compréhension de l'analyse classique de l'autoconfrontation croisée? C'est, selon Nadine Poussin, un moyen nécessaire pour comprendre comment se développe, voire se régule, un sentiment envers le travail et entre collègues. La dimension affective est ainsi appréhendée par la sélection d'un "moment clé », c'est-à-dire quand au moins deux des trois modalités deviennent pertinentes. Cette contribution apporte un «regard outillé » qui passe par la transcription de l'analyse qualitative des affects. Le dépouillement de ces moments se révèle comme un moyen d'appréhender les échanges qui ont suivi ces séquences afin de vérifier s'ils amenaient «une activité renouvelée de pensée », cela permettant de développer le pouvoir d'agir des professionnels enquêtés.

Albena Tcholakova apporte des éléments sur la place occupée dans l'enquête par les affects en partant du constat que tout travail engage des émotions de différentes natures. C'est à partir d'entretiens biographiques menés auprès de réfugiés dans deux pays (Bulgarie et France) que l'auteure explore les émotions dans le travail d'enquête. Elle s'interroge sur la «constitution émotionnelle» de l'engagement dans le travail d'enquête: comment les enquêtés rendent-ils compte de leurs émotions? Quels sont les moyens pour le chercheur d'avoir accès à ces émotions? Comment restituer cette réalité émotionnelle? En s'intéressant à la nature et aux effets de la dimension affective en lien avec un rapport de classe, de sexe et de racialisation, Albena Tcholakova rend compte de la dynamique interactionnelle enquêteurs-enquêtés. Ce faisant, elle s'interroge aussi sur la place de ses affects auprès de cette population migrante : jusqu'où aller dans l'empathie? Comment faire avec des enquêtés méfiants quant au rôle "d'espionne» de la chercheuse ou, au contraire, provoquer une distance en raison de tentatives insistantes de séduction, amenant l'auteure à mentir sur son statut matrimonial, utilisant même une alliance factice? Comment réaliser le travail émotionnel 
indispensable à la relation d'enquête, de surcroît lorsqu'on évoque des expériences traumatiques comme l'exil?

Au-delà du travail de terrain, l'article de Consuelo Vasquez s'intéresse à la pratique réflexive et critique de l'écriture ethnographique et sonde la manière de «s'y prendre pour (d)écrire les affects» ou interroger la graphie de l'ethnographie des affects. C'est sur deux terrains de recherche auprès d'un organisme de lutte contre le cancer (le premier porte sur la prévention et le second sur un événement de collecte de fonds), que la chercheuse, mais également bénévole et proche aidante, mobilise l'approche narrative de la critique habitée empruntée à Aschcraft pour faire apparaître elle. Consuelo Vasquez utilise les affects comme point de départ de la réflexivité et permet par la suite de dépasser la dualité objet/sujet. Dans cette approche, la mise en récit des affects suggère une «forme d'écriture performative » qui passe selon l'auteure par le fait de s'immerger dans l'expérience et se laisser affecter, de se placer au milieu, d'incorporer et incarner les affects et de dévoiler l'objet critique.

Dans sa contribution, Zoé Yadan montre comment elle a fait abstraction des dimensions affectives dans l'analyse de l'expérience vécue par les parents lorsque ceux-ci sont convoqués par le collège afin de répondre d'un «manquement grave» au règlement de leur enfant. La mise à distance des émotions sur son terrain de recherche, alors qu'elles étaient rétrospectivement, selon ses propos, « ostensibles » et « audibles », était une manière de répondre à l'injonction du travail d'objectivation des discours des parents lors des entretiens et de leurs actions observées pendant les conseils de discipline. C'est au moment de l'analyse des données, plus particulièrement lors de la retranscription des entretiens et d'un retour sur les notes de terrain, que les émotions sont devenues une clé de lecture pour appréhender les pratiques de ces parents en lien avec les normes de «bonne parentalité ». Cependant, en plus de la «neutralité axiologique » enseignée, elle souligne la difficulté en tant que jeune chercheuse à (dé)coder les émotions. Et c'est par le recours à un « portrait émotionnel» d'une mère de famille (au sens lahirien) que Zoé Yadan appréhende la dimension affective - réactions spontanées, réflexivités, difficultés d'expression, etc. - comme l'une des raisons des actions parentales.

Enfin, Anne Perriard, Carole Christe, Cécile Greset et Micaela Lois relatent l'expérience de différentes séances d'un atelier méthodologique. Dix-huit étudiantes inscrites au master en Études genre à l'Université de Genève et leur enseignante s'intéressent aux apprentissages de la sexualité en réalisant chacune deux entretiens biographiques auprès de différents publics et en élaborant des cartes cognitives pour illustrer les récits de vie. Le travail de 
groupe fait prendre conscience aux enquêtrices de la place des affects aux différentes étapes de l'enquête : avant, pendant et après l'entretien. De même, l'expérience collective lors des différentes séances de l'atelier fait ressortir le double intérêt de l'attention aux émotions (par leur verbalisation et leur partage) dans la production du savoir et dans l'apprentissage du métier de sociologue (et plus largement du point de vue pédagogique). À travers l'épistémologie du point de vue et le concept de travail émotionnel, cet article met également en lumière les enjeux de la recherche liés aux affects qui vont à l'encontre de l'idéal type du chercheur, soit l'enquêteur sans doute avant le terrain, sans sentiment de peur dans le choix des mots pour les relances et sans ressenti lors de la retranscription de l'entretien.

\section{Notes}

${ }^{1}$ Le terme affects est dans cette introduction employé dans son acception la plus générale, car renvoyant à un ensemble d'états affectifs : des émotions aux sentiments. Chaque contribution du numéro développe ses propos selon une terminologie singulière : affect, émotion, sensibilité, etc.

${ }^{2}$ Ces questions furent à l'origine de nos réflexions et de l'appel à contribution pour ce dossier. Parallèlement à cette démarche, quelques pistes ont été suggérées à ce sujet (Bonnet \& Rochedy, sous presse).

\section{Références}

Bernard, J. (2014). Une histoire de la sociologie des émotions? Dans F. Fernandez, S. Lézé, \& H. Marche (Éds), Les émotions. Une approche de la vie sociale (pp. 7-29). Paris : Éditions des archives contemporaines.

Bernard, J. (2017). La concurrence des sentiments. Paris : Métailié.

Bizeul, D. (2007). Que faire des expériences d'enquête? Revue française de science politique, 57(1), 69-89.

Bonnet, T., \& Rochedy, A. (sous presse). Regard croisé sur l'épreuve affective du terrain. Analyse des affects et chercheur.e.s affecté.e.s. Dans S. Héas, \& O. Zana (Éds), Les émotions du chercheur. Rennes : Presses universitaires de Rennes.

Braud, P. (1996). L'émotion en politique. Paris : Presses de Sciences Po. 
12 ReChERChES QuALitATIVES / Vol. 39(2)

Clough, P. T., \& Halley, J. (2007). The affective turn. Theorizing the social. Durham, NC : Duke University Press.

Corbin, A., Courtine, J.-J., \& Vigarello, G. (Éds). (2016a). Histoire des émotions (Tome 1). De l'antiquité aux lumières. Paris : Seuil.

Corbin, A., Courtine, J.-J., \& Vigarello, G. (Éds). (2016b). Histoire des émotions (Tome 2). Des lumières à la fin du XIX ${ }^{e}$ siècle. Paris : Seuil.

Corbin, A., Courtine, J.-J., \& Vigarello, G. (Éds). (2017). Histoire des émotions (Tome 3). De la fin du XIX siècle à nos jours. Paris : Seuil.

Cuin, C.-H. (2001). Émotions et rationalité dans la sociologie classique : les cas de Weber et Durkheim. Revue européenne des sciences sociales, 120(39), 77-100.

Damasio, A. R. (1995). L'erreur de Descartes : La raison des émotions. Paris : Odile Jacob.

Deluermoz, Q., Dodman, T., \& Mazurel, H. (Éds). (2018). Controverses sur l'émotion. Neurosciences et sciences humaines. Sensibilités. Histoire, critique et sciences sociales, (5).

Deluermoz, Q., Fureix, E., Mazurel, H., \& Oualdi, M. (2013). Écrire l'histoire des émotions: de l'objet à la catégorie d'analyse. Revue d'histoire du XIX $X^{e}$ siècle, (47). https://doi.org/10.4000/rh19.4573

Devereux, G. (1980). De l'angoisse à la méthode dans les sciences du comportement (trad. H. Sinanceur). Paris : Aubier. (Ouvrage original publié en 1967).

Durkheim, E. (1894). Les règles de la méthode sociologique. Repéré à http://classiques.uqac.ca/classiques/Durkheim_emile/regles_methode/durk heim_regles_methode.pdf

Ehrenberg, A. (2018). Figures de l'homme fiable ou l'esprit social des neurosciences cognitives. Sensibilités. Histoire, critique et sciences sociales, (5), 38-49.

Elias, N. (1993). Engagement et distanciation (trad. M. Hulain). Paris : Fayard. (Ouvrage original publié en 1983).

Febvre, L. (1941). La sensibilité et l'histoire: comment reconstituer la vie affective d'autrefois? Annales d'histoire sociale (1939-1941), 3(1-2), 5-20.

Ghasarian, C. (1997). Les désarrois de l'ethnographe. L'Homme, (143), 189198. 
Hochschild, A. (1983). The managed heart. Commercialization of human feeling. Berkeley, CA : University of California Press.

Kuhn, T. (2018). La structure des révolutions scientifiques. Paris : Flammarion.

Ledoux, J. (2005). Le cerveau des émotions. Les mystérieux fondements de notre vie émotionnelle. Paris : Odile Jacob.

March, J. G., \& Simon, H. A. (1999). Les organisations : problèmes psychosociologiques (trad. J. C. Rouchy et G. Prunier). Paris : Dunod. (Ouvrage original publié en 1958).

Mauss, M. (1921). L'expression obligatoire des sentiments (rituels oraux funéraires australiens). Journal de psychologie, (18), 425-434.

Mendras, H., \& Étienne, J. (1996). Les grands auteurs de la sociologie: Tocqueville, Marx, Durkheim, Weber. Paris : Hatier.

Montandon, C. (1992). La socialisation des émotions : un champ nouveau pour la sociologie de l'éducation. Revue française de pédagogie, (101), 105-122.

Olivier de Sardan, J.-P. (2000). Le «je» méthodologique. Implication et explication dans l'enquête de terrain. Revue française de sociologie, 41(3), 417-445.

Peneff, J. (2009). Le goût de l'observation: comprendre et pratiquer l'observation participante en sciences sociales. Paris : La Découverte.

Traïni, C., \& Siméant, J. (2009). Pourquoi et comment sensibiliser à la cause? Dans C. Traïni (Éd.), Émotions ... mobilisation! (pp. 11-34). Paris : Presses de la Fondation nationale des Sciences Politiques.

Villani, M., Poglia Mileti, F., Mellini, L., \& Singy, P. (2014). Les émotions au travail (scientifique) : enjeux éthiques et stratégies méthodologiques d'une enquête en terrain intime. Genre, sexualité \& société, (12). https://doi.org/10.4000/gss.3333

Weber, M. (1965). Essais sur la théorie de la science. Quatrième essai: "Essai sur le sens de la "neutralité axiologique" dans les sciences sociologiques et économiques» (trad. J. Freund). Paris : Plon. (Ouvrage original publié en 1917).

Thomas Bonnet est sociologue. Maître de conférences à l'Université Lyon 2 et chercheur au Centre Max Weber (CNRS UMR 5283), il travaille sur la thématique du lien entre travail, santé et émotions, notamment du point de vue des risques professionnels. Il a réalisé une recherche en partenariat avec l'INRS sur les questions 
14 ReChERChes QuAlitatives / Vol. 39(2)

des cultures organisationnelles de prévention dans le milieu de l'aide à domicile. Il a publié La régulation sociale du risque émotionnel au travail (2020) aux Éditions Octarès.

Amandine Rochedy est sociologue postdoctorante au Centre d'étude et de recherche travail, organisation, pouvoir (CERTOP, UMR CNRS 5044) à l'Université Toulouse Jean Jaurès. Elle travaille notamment sur la socialisation alimentaire des enfants en population spécifique (autisme, syndrome de Prader-Willi) et sur les pratiques alimentaires en population générale (primoparentalité, retraité). Ses travaux portent sur les constructions du répertoire alimentaire, entre normal et pathologique, et sur leurs gestions familiales au quotidien.

Pour joindre les auteurs :

t.bonnet@univ-lyon2.fr

amandine.rochedy@univ-tlse2.fr 